Evans, M. (2002), "FX Trading and Exchange Rate Dynamics". Journal of Finance 57 (6): 2405-2447.

Evans, M., y R. Lyons (2002), “Order Flow and Exchange Rates Dynamics”. Journal of Political Economy 110 (1): 170-180.

— $\mathrm{y}$ - (2004 a), "A New Micromodel of Exchange Rate Dynamics". NBER Working Paper 10379. Cambridge, Mass.

—, $\mathrm{y}-(2004 \mathrm{~b})$, "Frequently Asked Questions About the Micro Approach to FX”. Documento disponible en: http://faculty.haas.berkeley.edu/lyons.

Frankel, J., y A. Rose (1995), "Empirical Research on Nominal Exchange Rates". En G. Grossman, y K. Rogoff (eds.), Handbook of International Economics, vol.3. Amsterdam: Elsevier/North Holland.

Flood, R., y M. Taylor (1996), "Exchange Rate Economics: What's Wrong with the Conventional Macro Approach”. En J. Frankel, G. Galli y A. Giovannini (eds.). The Microstructure of Foreign Exchange Markets. Chicago: University of Chicago Press.

Froot, K. A., y T. Ramadorai (2005), "Institutional Portfolio Flows and International Investments". Review of Financial Studies (en prensa)

Kennedy, P. (2003), A Guide to Econometrics. $5^{\mathrm{a}}$ ed. Cambridge: The MIT Press.

Klitgaard, T., y L. Weir (2004), "Exchange Rates Changes and Net Position of Speculators in the Futures Market”. FRBNY Economic Policy Review 10 (1): 17-28.

Lyons, R. (2001), The Microstructure Approach to Exchange Rates. Cambridge: The MIT Press.

Meese, R., y K. Rogoff (1983), "Empirical Exchange Rate Models of the Seventies: Do They Fit Out of Sample?". Journal of International Economics 14(2): 3-24.

Payne, R. (2003), "Informed Trade in Spot Foreign Exchange Markets: An Empirical Investigation”. Journal of International Economics 61 (2): 307-329.

Torre, L., y O. Provorova (2007), "Tipo de cambio, posiciones netas de los especuladores y el tamaño del mercado de futuros del peso mexicano". Economía Mexicana Nueva Época XVI (1): 5-46.

\section{Gasto en desarrollo social en el contexto del modelo neoliberal en México 1995-2007: la reorientación del papel del Estado}

José Luis Martínez Marca

RESUMEN

La política pública de gasto en desarrollo social ha tomado relevancia en los últimos años en varios países de América Latina y, en particular, en México. Esta importancia se asocia con la creciente desigualdad social y la concentración de la riqueza, resultado de la aplicación de las políticas neoliberales desde inicios de la década de los ochenta en México.

El objetivo fundamental de este artículo es identificar los principales cambios que se han realizado en materia de política pública de gasto social en México, mediante un análisis comparativo entre el tipo y el monto del gasto en desarrollo social en dos periodos. El primero comprende de 1970 a 1982, durante el fin del modelo de sustitución de importaciones. El segundo corresponde a análisis del gasto social ejercido por el gobierno mexicano en el contexto del modelo neoliberal, después de la crisis financiera de México en 1995.

Número de clasificación: JEL: H53

Palabras clave: política pública de gasto social, teoría poskeynesiana, política neoliberal, reorientación del papel del Estado.

\section{Abstract}

The public social development expenditure policy has been enforced in the last years in severa countries of Latin America and particularly in Mexico. These tendencies have been associated to social inequality and the concentration of wealth, as a result of neo-liberal policies applied since the beginning of the seventies in our country.

In this sense this article aims to identify the main changes which have been carried out in the public social development expenditure policy in Mexico, by means of a comparative analysis of the type and amount of the social development expenditure during two periods.

The first one spans from 1970 to 1982, during the end of the imports substitution pattern. The second period involves the analysis of the social expenditure made by the Mexican government in the neo-liberal pattern framework, after the 1995 financial crisis in Mexico, through 2008.

Number of classification: JEL: H53

Key words: Public social development expediture policy, neo-liberal policy, new axe state role.

"Profesor-investigador de tiempo completo del posgrado en Economía, Facultad de Estudios Superiores Aragón de la Universidad Nacional Autónoma de México [joslumm21@ hotmail.com]. 


\section{INTRODUCCIÓN}

La importancia de la política pública de gasto en desarrollo social se ha incrementado en los últimos años en varios países de América Latina, y particularmente en México. La creciente desigualdad social y la concentración de la riqueza registradas en décadas pasadas, asociadas a la aplicación de las políticas neoliberales desde los años ochenta, son factores que han incidido en la necesidad de fomentar políticas de gasto social para estimular el desarrollo del país.

La expansión del gasto en desarrollo social se ha convertido en un instrumento de las políticas públicas por parte del Estado para reducir los niveles de pobreza y desigualdad, acentuados por la aplicación de políticas macroeconómicas. ${ }^{1}$ No obstante, las políticas de gasto social estarán acotadas por los límites que las políticas neoliberales imponen para mantener el equilibrio financiero del sector público y, con ello, la estabilidad en los precios. Así, los alcances de la política de gasto social en la economía mexicana se verán mermados y, en consecuencia, se vislumbra una tendencia a aumentar las brechas de pobreza y desempleo. La creciente migración de la población rural a las grandes ciudades del país y hacia Estados Unidos es una expresión de la agudización de la pobreza y de la falta de oportunidades laborales y de mejoría del ingreso.

El objetivo central de este artículo es identificar y analizar cuáles son los principales cambios que se han realizado en la política pública de gasto social en México. El estudio se realiza a partir de un análisis comparativo de dos periodos entre el tipo y monto del gasto en desarrollo social realizado. El primero corresponde a la última fase del modelo de sustitución de importaciones de México, de 1970 a 1982. El segundo periodo que se estudia es el posterior a la crisis de 1995 , en el contexto de la aplicación de las políticas neoliberales.

Junto al análisis comparativo del gasto social ejercido en México según los dos modelos de crecimiento mencionados, se reflexiona sobre la necesidad de reorientar el papel del Estado, con un enfoque teórico poskeynesiano. En especial, este tema adquiere relevancia frente a los graves efectos de desarrollo social que detectan las economías emergentes, asociados a la aplicación de las políticas neoliberales. En este contexto, nos preguntamos si existen diferencias sustantivas en la naturaleza de las políticas sociales aplicadas en los dos modelos de desarrollo y si se considera pertinente continuar con una política de gasto social en la que prevalecen las políticas neoliberales y el reducido crecimiento eco-

${ }^{1}$ En efecto, en las grandes metrópolis, pero también en el país en su conjunto, la pobreza creció sustantivamente y, asociada a ella, se detecta un aumento considerable en los índices de delincuencia. nómico. Aunque esta investigación no se propone evaluar la eficiencia de los dos modelos de gasto social, la disminución de los índices de pobreza y el incremento en el desarrollo social, se reconoce la necesidad de continuarla para tener resultados más contundentes.

\section{Principales aspectos conceptuales sobre el gasto público SOCIAL EN EL MODELO DE SUSTITUCIÓN DE IMPORTACIONES VS. EL MODELO NEOLIBERAL}

Desde 1939 hasta inicios de la década de los ochenta, el crecimiento de la economía mexicana se caracterizó por una amplia participación del Estado en la economía mediante la aplicación del gasto público. Fundamentalmente éste se orientó a la ampliación de la infraestructura y de las empresas públicas que se crearon con el fin de fortalecer el crecimiento industrial sustitutivo de importaciones. El Estado, a su vez, se caracterizó por ser protector, inversor y promotor del crecimiento de la economía. El enorme gasto público ejercido, en especial en los años setenta, superó significativamente los ingresos, dando como resultado la existencia crónica del llamado déficit financiero:

La transformación política y social de México a raíz de la Revolución y el proceso de crecimiento económico sostenido a partir de 1934 avalaban la favorable opinión que sobre el país tenían los inversionistas y los banqueros extranjeros, sobre todo cuando ese crecimiento se combinó con la estabilidad monetaria y la de precios después de 1954, y daban sólido sostén al razonado optimismo y a la confianza que sobre las posibilidades de desarrollo de México tenían los capitales nacionales [Tello, 2007, p. 452.]

La llamada transformación de la economía se basó justamente en la fuerte injerencia del Estado en la economía, con lo cual, al igual que en casi toda Latinoamérica, se seguían los preceptos derivados de la teoría de John M. Keynes. Se sugería que dadas las imperfecciones del mercado era necesaria la participación del Estado en la economía para hacer más eficiente el proceso económico y con ello lograr un proceso de crecimiento autosostenido de largo plazo, como lo proponían los preceptos establecidos en el modelo de sustitución de importaciones de aquella época.

De esta manera, la primera gran crisis económica del capitalismo en 1929 demostró que el mercado no era tan eficiente para la determinación de los precios y la asignación de los recursos como lo establecía la llamada teoría neoclásica. En 
ese sentido se dieron los elementos para fortalecer la posición keynesiana en términos de las imperfecciones del mercado y de la necesidad de intervención del Estado en la economía. Así, se tenía que:

en las economías realmente existentes casi nunca se cumplen los supuestos de la competencia perfecta y, por lo contrario, lo común es que los mercados no sean mecanismos tan perfectos como los que describe un modelo ideal. En general, los mercados presentan algunas imperfecciones, es decir, los mercados potencialmente pueden fallar en alcanzar los resultados eficientes y mucho menos llegan a resultados socialmente equitativos. Si los mercados fallan, entonces se abre una posibilidad para corregir estas fallas a través de la intervención del Estado [Tello, 2007, p. 452.]

Dada la ausencia de mercados perfectos, las fallas de éstos respaldan la participación del Estado en la economía. Éste fue uno de los principales factores que junto con las recomendaciones de política económica de Keynes, llevaron a que el Estado mexicano asumiera una importante participación en la economía durante el llamado modelo de sustitución de importaciones, que operó en México durante el periodo de 1939 a principios de la década de los ochenta, cuando se inicia un periodo de transición hacia el llamado modelo neoliberal, que se aplicaría a partir del gobierno de Carlos Salinas en 1989. Dicho modelo sustentaría entre sus preceptos fundamentales los lineamientos derivados del consenso de Washington de 1989, con una serie de reformas que se orientarían principalmente a la disminución de la participación del Estado en la economía y a mantener un equilibrio fiscal (Ibarra, 2006, p. 101), regresando a los viejos principios de la teoría neoclásica con algunas diferencias, entre las que sobresale el proceso de globalización de las economías.

Las reformas, el "paquete" de cambios, incluyen la liberación del comercio exterior, del sistema financiero y de la inversión extranjera. La orientación de la economía hacia los mercados externos. La privatización de las empresas públicas. La desregulación de las actividades económicas. La estricta disciplina fiscal. La erradicación de los desequilibrios fiscales, por la vía de la reducción del gasto público. Una reforma tributaria orientada a la reducción de las tasas en los impuestos directos y a descansar, para la recaudación, en los impuestos indirectos. Un marco legislativo e institucional para garantizar los derechos de propiedad. [Tello, 2007, p. 626.]
Se tiene un regreso claro por parte de los gobiernos a la puesta en práctica de la doctrina teórica neoclásica, con la que se reconocen las bondades del mercado. Aunque se está consciente de sus imperfecciones, se le permite al Estado participar en la economía sólo en aquellas actividades que para la iniciativa privada no representan ningún interés de negocio importante, pero se insistirá en la apertura de actividades que le son rentables, como ha sido el caso en México en agosto de 2008, a propósito de la participación del sector privado en la explotación del petróleo.

Bajo esta estructura de política económica global inserta en el marco del modelo neoliberal, es evidente que las políticas públicas que surgen - como es el caso de la de gasto para el desarrollo social en la economía - tenderán evidentemente a ser sólo un paliativo contra la desigualdad que genera la política económica neoliberal y nunca una solución, dado que el propio modelo lo establece de esa manera y se orienta principalmente a garantizar la rentabilidad del capital privado y asegurar los principios de la propiedad privada del mismo capital a fin de constituir un mercado atractivo para la inversión extranjera y nacional, dadas las características que la globalización impone a los países de la región latinoamericana.

Existe consenso entre un gran número de investigadores del desarrollo económico que reconocen que el modelo neoliberal que actualmente se ha aplicado en varias economías de América Latina no ha conducido a los resultados esperados, sobre todo para la gran mayoría de la población. Es el caso del maestro Carlos Tello (ex secretario de Hacienda en el periodo de gobierno del entonces presidente José López Portillo):

El modelo neoliberal que con entusiasmo adoptan los gobiernos, promete mejorar la competitividad, estimular el libre acceso a los recursos de capital y a los tecnológicos y promover una estructura económica más flexible. Todo ello para desarrollar un ambiente más favorable para los inversionistas nacionales y extranjeros y así lograr un crecimiento económico sostenido, estabilidad en los precios y los más altos niveles de bienestar. No ha sido el caso. La política adoptada de inmediato arrojó, como su primer resultado para las economías de la región latinoamericana, la llamada década perdida, que en realidad para muchos países resultaron ser dos décadas perdidas. [Tello, 2007, p. 626.]

De esta manera la propuesta de la reorientación del papel del Estado (Martínez Marca, 2006, p. 101) reside esencialmente en fortalecer el gasto público en su carácter anticíclico, mediante la aplicación de políticas públicas como 
la de desarrollo social e infraestructura con el fin de fortalecer el desarrollo de su mercado interno y promover el empleo. Esto produciría mejoras en el aumento de la demanda agregada y la reducción de los choques externos en México como la que se registró recientemente (segundo semestre de 2008), derivada de la desaceleración de la economía de Estados Unidos. Es necesario regular las actividades económicas, sobre todo la financiera y fiscal, a fin de promover un crecimiento del financiamiento a la inversión - visto desde la perspectiva poskeynesianaque mejore las condiciones de desarrollo social en el largo plazo en la región (Martínez Marca, 2007, p. 253).

\section{El GaSTO PÚBLICO EN DESARROLlo SOCIAL EN LA RUPTURA DEL MODELO DE SUSTITUCIÓN DE IMPORTACIONES EN MÉXICO 1970-1982}

Uno de los principales problemas que se manifestó en las economías latinoamericanas, y en particular en México, derivado de la estrategia de crecimiento económico basada en el modelo de sustitución de importaciones, fue el creciente endeudamiento externo que se registró en la economía mexicana durante el periodo de 1970 a 1982, y que impactaría de manera negativa el margen de maniobra del gasto público con fines de promoción del crecimiento, y en particular del gasto destinado al desarrollo social durante esa época. Una de las primeras manifestaciones de dicha reducción del margen de maniobra se presentó de manera clara en el fuerte crecimiento del déficit financiero del sector público.

Algunas cifras ilustran este proceso. Por ejemplo, conforme a las cifras de endeudamiento público externo, la deuda pasó de un nivel de 9,975 millones de dólares en 1974 a 58,874 millones de dólares para 1982, lo cual indicaba que en buena medida el fuerte gasto del gobierno era financiado por el endeudamiento público externo. Esto se volvió un lastre en el mediano plazo y también un factor de restricción más que de promoción del crecimiento económico, pues éste se registraba bajo el modelo de sustitución de importaciones que privilegiaba el gasto de inversión y social a fin de estimular el crecimiento del país. La situación se reflejaba en un fuerte déficit financiero que no era compensado con el aumento de los ingresos tributarios del Estado.

Uno de los objetivos del modelo de crecimiento se basaba principalmente en la participación del Estado en la economía como rector del crecimiento y asignador de recursos y también formador de precios. Ese fue el caso en la determinación de los precios de los bienes y servicios que eran producidos en ese momento por el sector público y que representaban al cierre de 1976 más de mil empresas públicas propiedad mayoritaria del Estado lo cual nos da una clara idea de la importancia que tenía el gobierno en la promoción de la producción en México durante ese periodo.

La creación de instituciones públicas, como el Instituto Mexicano del Seguro Social, el Instituto de Seguridad Social al Servicio del los Trabajadores del Estado, la Compañía de Subsistencias Populares (Conasupo), entre muchas más empresas, refleja la importancia que tenía el gasto social durante el periodo del llamado crecimiento sustitutivo de importaciones que estuvo presente en México hasta principios de la década de los ochenta, pero que, sin embargo, dio lugar al fuerte crecimiento del déficit financiero del sector público. Esto lo podemos apreciar en la gráfica 1.

GRÁFICA 1. Balance financiero del sector público en México (porcentaje del PIB)

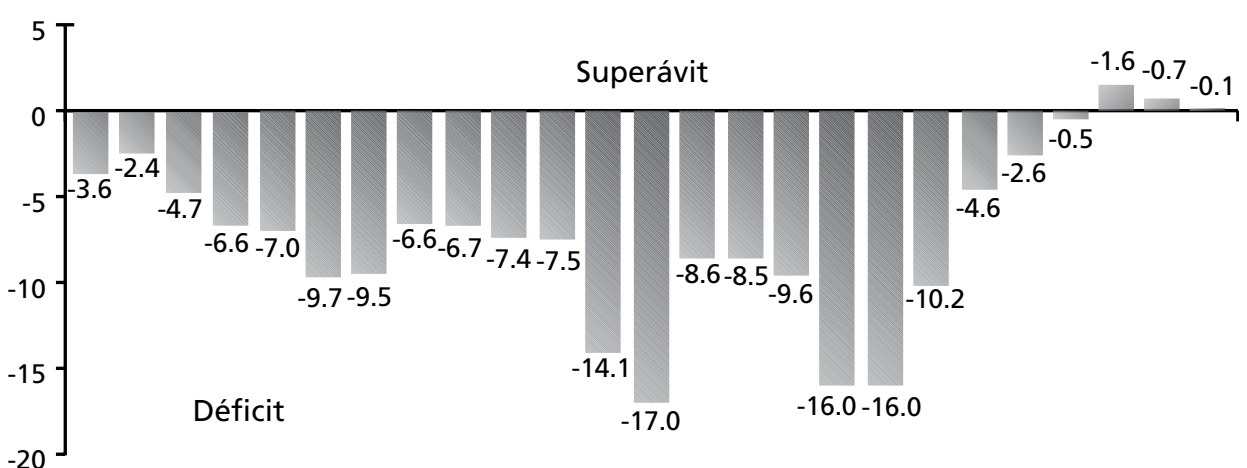

Fuente: Elaborada con base en datos de la SHCP.

De esta forma se señala que parte del agotamiento del modelo de sustitución de importaciones registrado a principios de la década de los ochenta es producto de la fuerte restricción presupuestaria que mantenía el sector público de la economía y que se reflejaba en un fuerte crecimiento del déficit financiero, dada la imposibilidad que se mantenía por elevar los ingresos públicos. Imposibilidad debida a la existencia de una política deliberada de subsidio al sector privado por medio de los precios de los bienes y servicios que producía el sector público con un alto impacto de difusión en el aparato industrial y de servicios, tales son los casos, por ejemplo, de la gasolina, luz eléctrica, transporte ferroviario, transporte marítimo, productos de consumo popular como la leche, el maíz, entre otros, lo cual evidentemente dio como resultado una fuerte presión sobre las finanzas públicas por el lado de los ingresos que tuvieron que ser compensados mediante 
un endeudamiento público externo para compensarlos, dado que el gasto no se redujo sustancialmente durante ese periodo. Tal es el caso del gasto destinado a los aspectos sociales como la salud, la seguridad pública, el transporte, la educación, la producción para la alimentación popular vía la Conasupo, que presionaron sin duda las finanzas públicas. Véase la gráfica 2.

Grafica 2. México: Gasto del sector público en desarrollo social (porcentaje del PIB)

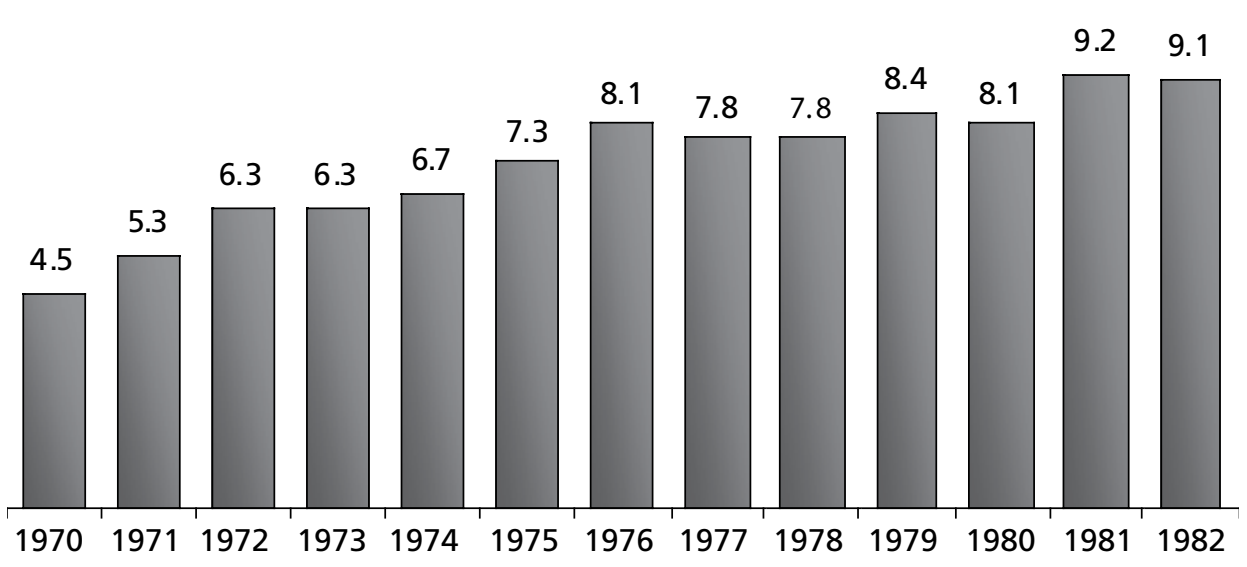

Fuente: Elaborada con base en datos de la SHCP.

Sin duda, la importancia del gasto en desarrollo social en México fue notoria de 1970 a 1982, dado el crecimiento que registró el mismo en ese periodo al pasar de un nivel de $4.5 \%$ en 1970 a casi el doble en 1982, cuando alcanzó un nivel de $9.1 \%$ respecto al PIB. Esto representa los niveles más altos registrados durante la vigencia del modelo de sustitución de importaciones, que se caracterizaba por una importante participación del Estado como inversor, promotor y protector del crecimiento de la economía mexicana y que suponía que en el largo plazo todo ello redundaría en un desarrollo social y despegue industrial de la economía tal y como había sido concebido a finales de la década de los años treinta.

Sin embargo, los impactos negativos que provocó el fuerte déficit financiero del sector público sobre la inflación y el tipo de cambio desde el inicio de la década de los setenta desembocaron en el cambio de las expectativas de los agentes económicos nacionales que, combinados con la existencia de un entorno internacional en donde se presentaba como un paradigma al neoliberalismo económico con una fuerte injerencia del pensamiento monetarista (que influía en el pensamiento económico de los organismos financieros internacionales), se concretaron en la economía mexicana: primero en las devaluaciones que el peso registró desde septiembre de 1976 y que continuaron durante casi toda la década de los ochenta; segundo, en el creciente aumento de la inflación, que minó el poder adquisitivo de los salarios y que deprimió el mercado interno, a la vez que incidía en la reducción de la oferta del empleo y el aumento de la inversión privada; en tercer lugar, en la creciente fuga de capitales, cuyo origen estaba determinado por los principales inversionistas nacionales tanto del sector financiero como industrial, lo cual aumentó la presión sobre el tipo de cambio (Girón González y Correa, 2002, p.12).

La combinación de los procesos anteriormente señalados obligaron al gobierno a modificar el modelo económico de sustitución de importaciones para retomar las recomendaciones de los organismos financieros internacionales desde 1983, y que serían caracterizados como el modelo neoliberal que abordaremos en el siguiente apartado y que tenía que ver con el mantenimiento de un equilibrio fiscal público que implicaría la reducción de la participación del Estado en la economía.

\section{El gasto Público SOCIAL EN EL CONTEXTO DEL} MODELO NEOLIBERAL EN MÉXICO, 1995-2007

La fuerte influencia que ejercieron en los gobiernos de América Latina los organismos financieros internacionales como el Fondo Monetario Internacional y el Banco Mundial durante la década de los ochenta respecto a la política económica a seguir (cuya recomendación giraba en torno a la adopción de un modelo de crecimiento basado en la apertura de la economía y a la disciplina fiscal del Estado, y que fue institucionalizado de alguna manera con el Consenso de Washington en 1989) afectaría de manera significativa el papel que el Estado había tenido durante el modelo de sustitución de importaciones respecto al nuevo modelo que se definiría como neoliberal y que estaría caracterizado por una fuerte disminución de su injerencia en la economía.

\section{La política monetaria}

Se obligó al mantenimiento de una estricta disciplina fiscal con fines de estabilización de precios y del tipo de cambio, bajo lineamientos de corte monetarista que actuarían sobre la base monetaria y, por tanto, sobre el crédito interno (véase gráfica 3) y la tasa de interés del mercado interno, con lo cual se limitaba el crecimiento de la demanda agregada y, en consecuencia, el potencial de crecimiento en la economía. 
GráfICA 3. Crédito interno neto

(Miles de millones de pesos de junio de 2002)

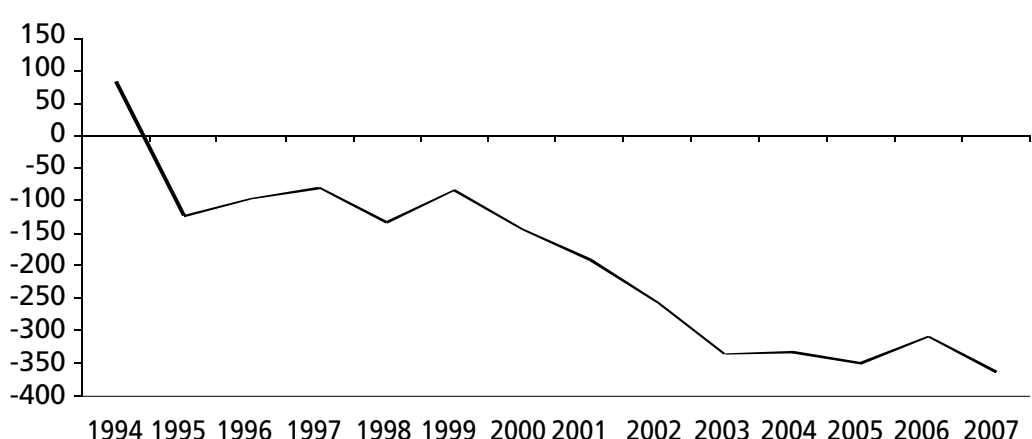

Fuente: Elaborada con base en datos del Banco de México.

Que se mantenga un control estricto de la base monetaria en la economía mexicana demuestra aspectos muy importantes. El primero de ellos se refiere, en efecto, a que el objetivo central del Banco de México para lograr la estabilización de precios y del tipo de cambio - después de la devaluación de diciembre de 1994 y que se manifestó en la crisis financiera de 1995- se basa en su totalidad en un esquema de corte monetario propuesto por el modelo neoliberal. Dicho esquema consiste en atacar la inflación por el lado de la demanda promoviendo el control en el aumento de la base monetaria respecto a los aumentos en los activos internacionales netos. Con esto se promueve una contracción en el circulante y por consecuencia se manifiesta en un aumento negativo en el crédito interno neto de la economía, es decir, una restricción en el circulante mediante la emisión de valores públicos que da lugar al aumento en rendimiento real de los mismos, lo cual afecta sin duda el crecimiento de la inversión productiva y por consecuencia del empleo, impactando negativamente la perspectiva de crecimiento económico a costa de la estabilidad de precios, lo cual beneficia la rentabilidad del capital financiero.

Lo anterior se complementaría mediante la restricción al gasto público por parte del Estado a fin de reducir las presiones de demanda sobre el crédito interno $y$, en consecuencia, sobre la base monetaria. Lo que supone el modelo neoliberal, entonces, es que la estabilidad de precios en la economía se convierte en un fin en sí mismo. De esta manera, la autoridad monetaria - en este caso, el Banco de México - se comprometerá a sostener y mantener una política monetaria restrictiva que garantice la estabilidad de los precios y del tipo de cambio, promoviendo una política de tasas de interés reales positivas que hagan atractivo el mercado financiero de México y estimulen el crecimiento de los activos internacionales (véase la gráfica 4).
GrÁFICA 4. Base monetaria y activos internacionales (Miles de millones de pesos de junio de 2002)

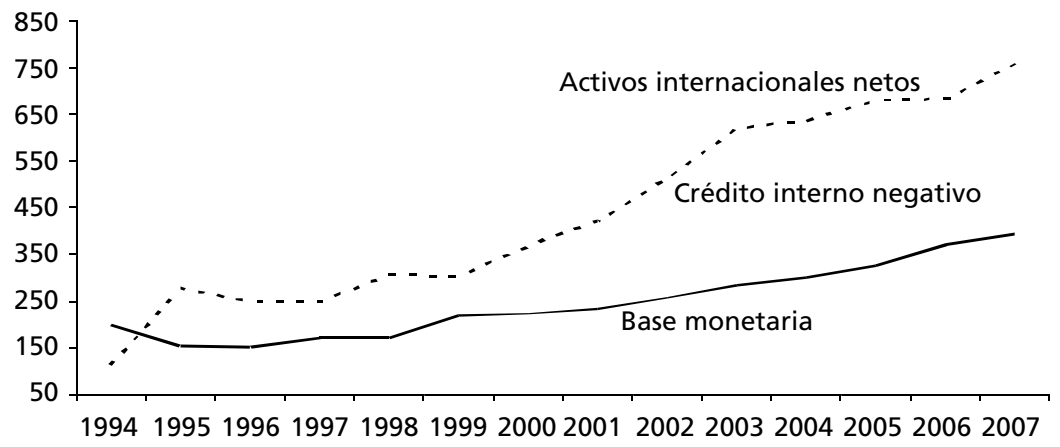

Fuente: Elaborada con base en datos del Banco de México.

\section{La política de gasto público}

El proceso de ajuste monetario se manifestaría en un cambio drástico sobre el balance financiero del sector público con la aplicación del modelo neoliberal, el cual desde principios de la década de los noventa empieza a mostrar incluso superávit. Posteriormente se mantendrá una política estricta de equilibrio entre los gastos e ingresos del sector público, permitiéndose sólo niveles de déficit que no excedan en $1.5 \%$ respecto al PIB y no presionen el crecimiento de la base monetaria. Esto vuelve restrictiva la política monetaria y convierte al banco central en el eje de la política económica actual en México (véase la gráfica 5).

\section{GRÁFICA 5. Déficit financiero del sector público} (Porcentaje del PIB)

10.2

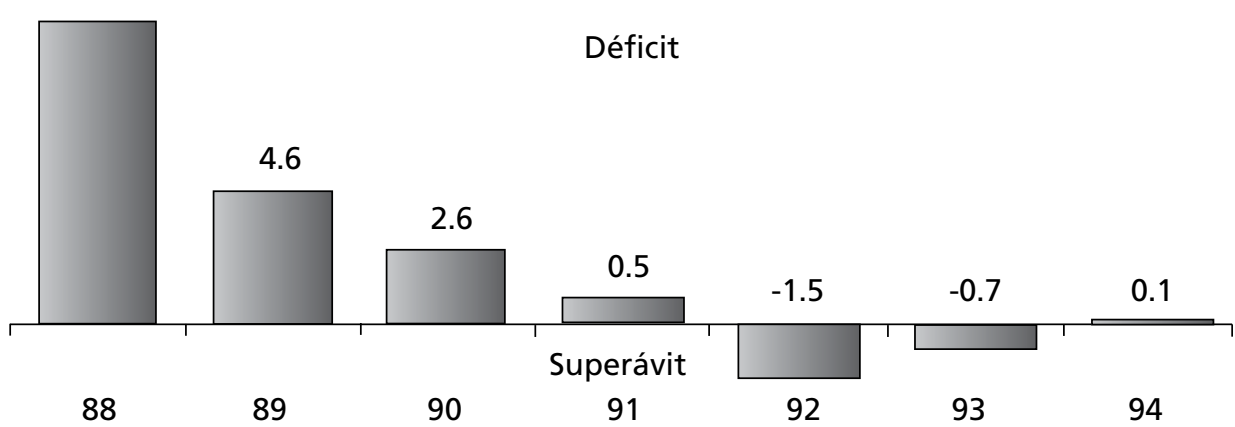

Fuente: Elaborada con base en datos de la SHCP. 
Con dicho proceso, al final de cuentas se establece entonces que la participación del Estado en la economía pone en riesgo la estabilidad macroeconómica, dado que presiona la demanda de liquidez en la economía, y por consecuencia los esfuerzos del banco central por estabilizar el crecimiento de dicha liquidez. Dicho planteamiento coincide con los postulados monetaristas que fueron recomendados mediante el Consenso de Washington desde 1989 y que suponen entonces que:

Las conclusiones básicas del monetarismo son:

- La eficiencia de la política fiscal para afectar la demanda agregada depende decisivamente de la política monetaria. Una política monetaria que tienda a neutralizar la velocidad de circulación del dinero hará completamente ineficaz la política fiscal y, en contraste, una política monetaria que estabilice las tasas de interés hace que la política fiscal influya decisivamente en la demanda agregada.

- Subraya la necesidad de una política económica pasiva y una reducida intervención estatal. La intervención del Estado debe concentrarse en tres áreas: protección de los derechos individuales; arbitraje en las disputas en el intercambio de derechos de propiedad por medio de reglas claras, y corrección de las fallas del mercado, pero en un marco de libertad. En cambio destacan las bondades del mecanismo de los precios para alcanzar el equilibrio económico.

- Critica las políticas fiscales expansivas por sus secuelas inflacionarias y desestabilizadoras, $y$

- Reduce la importancia de cumplir metas orientadas a alcanzar el pleno empleo y en cambio acentúa la importancia de mantener la estabilidad macroeconómica como condición indispensable para alcanzar el pleno empleo [Ayala Espino, 2004, p. 215.]

El comportamiento de la política monetaria que se expuso anteriormente toma como base el manejo de la base monetaria en México y, por tanto, el comportamiento del crédito interno que es negativo, es decir, que el crecimiento de los activos internacionales es mayor que el de la base, demuestra la evidente política monetaria restrictiva con fines de estabilización de precios, lo cual confirma el carácter ortodoxo de la política monetaria que es aplicada en México al menos desde 1995, fecha en que se presentó la crisis financiera en nuestro país.

De esta manera, uno de los elementos que complementa el objetivo de estabilización de los precios es justamente el equilibrio de las finanzas públicas, el cual se logra principalmente mediante la reducción de la participación del Estado en la economía. Por tanto, se observa en la reducción del déficit financiero del sector público y en consecuencia en la baja de su gasto público, en donde encontramos al gasto dirigido al desarrollo social, tal y como se puede apreciar en la gráfica 6 .

GRÁfICA 6. Gasto público en desarrollo social: México (Porcentaje respecto al total del gasto público)

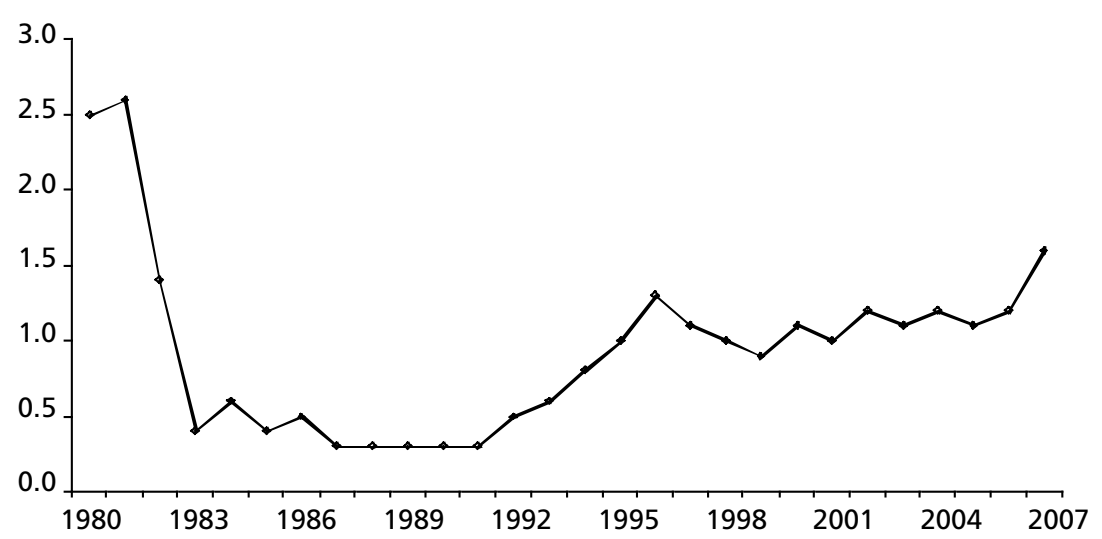

Fuente: Elaborada con base a datos de la SHCP.

En este sentido, se puede desprender como dichas políticas que postulaban como uno de sus mayores méritos, la reactivación acelerada del crecimiento económico, sin embargo, a medida que avanza el modelo neoliberal se observa un deterioro en los indicadores económicos en términos de su tasa de crecimiento, e incluso del deterioro del gasto social con fines de abatimiento de la propia pobreza que genera la aplicación del modelo neoliberal. Así tenemos que las magnitudes de los retrocesos se manifiestan en:

- Reducción del PIB.

- Pérdida del poder adquisitivo.

- Descenso drástico de los salarios reales.

- Disminución de los niveles de empleo.

- Concentración más aguda del ingreso.

- Reducción del gasto público en servicios sociales, lo que deteriora el salario indirecto.

- Concentración de los medios de producción. 
GRáfICA 7. Gasto público en desarrollo social y superación de la pobreza

(Porcentaje respecto al total del gasto público)

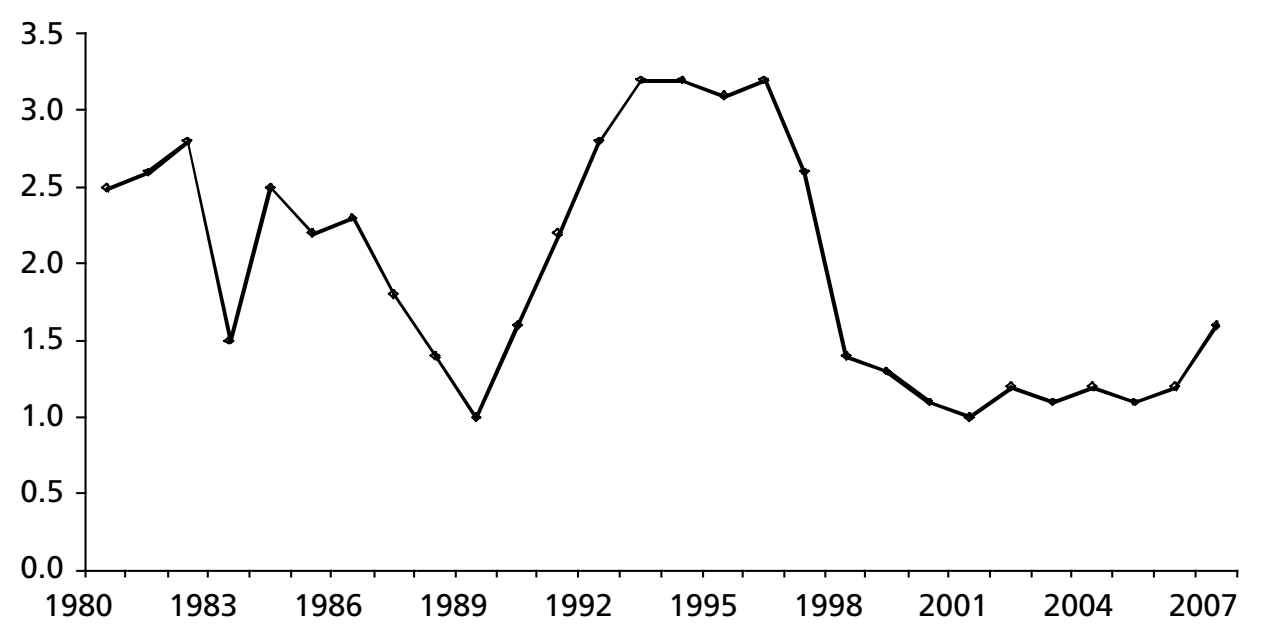

Fuente: Elaborada con base en datos de de la SHCP.

\section{Debilitamiento de los productores medianos y pequeños ante} las empresas transnacionales

El costo social del funcionamiento de las políticas neoliberales es elevado, no obstante que sus principales defensores estipulaban que las políticas neoliberales generarían los recursos suficientes para compensar los costos sociales. Sin embargo, a dos décadas de la instrumentación de dichas políticas en México, se observa que lejos de crear los recursos necesarios para compensar los costos sociales, se ha agudizado la dimensión de esos costos, viéndose reflejada en: incremento de la pobreza, deterioro en las condiciones de vida de la mayor parte de la población, contracción de los salarios reales, pérdida de empleos y polarización del ingreso, como se muestra en la gráfica 7 , en donde se observa una caída de los gastos relacionados con el desarrollo social y superación de la pobreza en los últimos años en México.

Las consecuencias sociales (las expresiones no económicas de la pobreza) no se han hecho esperar y se manifiestan en los siguientes fenómenos: creciente extensión del comercio de menores, incremento de la delincuencia y exacerbación de los niños de la calle.

Las medidas tomadas por el Estado relacionadas con la política neoliberal impactan de manera negativa en el gasto destinado al desarrollo social, y si con- sideramos que la economía de mercado es desigual, dicha discrepancia se profundiza aún más.

Es importante señalar cómo, en efecto, los requerimientos de estabilidad de precios han sido complementados en México por el ajuste de sus finanzas públicas. Dicho ajuste se hace sobre todo a partir de la contracción del gasto público, afectando renglones no sólo de inversión en infraestructura que son necesarios para mejorar las condiciones de competencia de la economía mexicana, sino también a las tasas de crecimiento de los empleos en nuestro país, tal como se puede apreciar en la gráfica 8 , en donde se observa que durante el siglo XXI la tasa de crecimiento de los asegurados permanentes en el Instituto Mexicano del Seguro Social - que registra los empleos permanentes que son creados en la economía - se ha reducido de manera significativa con el crecimiento que reconocen a final de la década de los noventa.

Asimismo, debemos destacar que en México la participación del gasto público en desarrollo social en la actualidad ha perdido importancia de manera significativa, con lo cual podemos afirmar que los esfuerzos de la política pública se han centrado en mantener la estabilidad de precios, soslayando el apoyo a los sectores más desfavorecidos de la población, ampliándose la brecha de desigualdad que genera el propio modelo neoliberal y la globalización de la economía.

\section{GráfICA 8. Asegurados permanentes en el ImSS}

(Variación porcentual anual)

enero 1995-agosto 2005

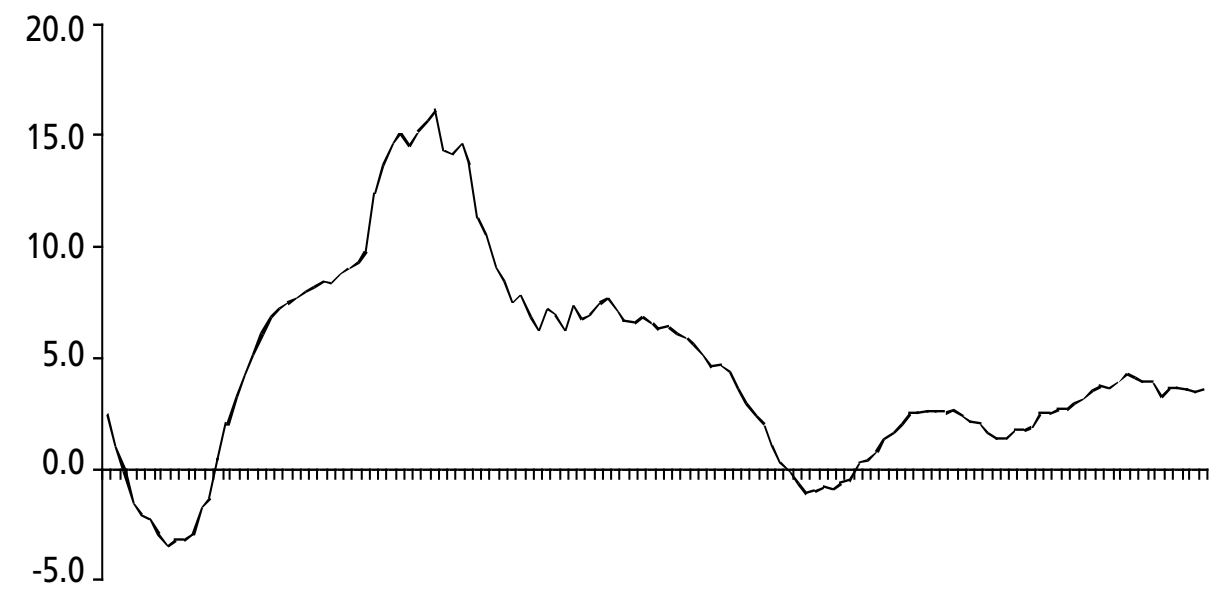

1995 J 1996 J 1997 J 1998 J 1999 J 2000 J 2001 J 2002 J 2003 J 2004 J 2005 J

Fuente: Elaborada con base en datos del IMss.

*Respecto al crecimiento total. 


\section{Principales cambios y Perspectiva}

\section{DEL GASTO PÚBLICO SOCIAL EN EL FUTURO INMEDIATO}

Se ha señalado que uno de los pilares de la política neoliberal es la reducción de la participación del Estado en la economía, incluyendo no sólo su actividad promotora en la inversión, sino también una reducción en su gasto. La implantación de dichas políticas tiene como objetivo la consecución de balanzas públicas equilibradas (equilibrio fiscal) y la estimulación de la estabilidad de precios. En México, para poder alcanzar un presupuesto equilibrado, ${ }^{2}$ se han instrumentado políticas tendientes a aumentar el ingreso y disminuir el gasto público. En cuanto al ingreso público, las políticas han girado en torno a aumentos de los impuestos indirectos, mientras que en el gasto se han aplicado políticas de recortes que han sido evidentes en el gasto de inversión, infraestructura y social.

Las políticas neoliberales buscan alcanzar en equilibrio fiscal debido a que se considera que el gasto público produce un efecto de crowding out en el gasto privado, lo que genera una elevación en las tasas de interés, que propicia una apreciación de la moneda y conduce a su vez a un aumento del déficit comercial, ocasionando una mayor presión inflacionaria. Por tal razón, en el modelo neoliberal se considera que un déficit fiscal implica presiones inflacionarias y déficit comercial, lo cual va en contra de los objetivos de estabilidad de precios, finanzas públicas equilibradas y balanza comercial externa equilibrada.

Por ello, si México sigue los lineamientos del modelo neoliberal, es de esperarse que para el futuro inmediato la política de gasto público siga los mismos lineamientos de austeridad, con reducciones importantes en el gasto social, que recientemente incluye el orientado a la reducción de la pobreza, de desarrollo y de inversión, lo que genera menor crecimiento, mayor desempleo, mayor desigualdad del ingreso y pobreza.

Según este escenario resulta importante establecer otro planteamiento de política económica que permita alcanzar mayor crecimiento y empleo y menor pobreza. La mayoría de las propuestas de política económica consideran relevante la reorientación de la función del Estado - que más adelante se expone de manera amplia - . Si bien para los poskeynesianos el desempeño del Estado como motor de crecimiento es importante, cabe señalar que dependiendo de la vertiente poskeynesiana que se considere, la función del Estado en la consecución de un

${ }^{2}$ El planteamiento ortodoxo considera que un déficit fiscal implica un déficit comercial, ya que un déficit fiscal eleva la tasa de interés y con ello la apreciación de la moneda. Para mayores referencias véase: Wray, (2000, 2005, 2006, 2006 a y 2007). mayor crecimiento será principal o secundaria. Para la vertiente horizontalista, ${ }^{3} \mathrm{el}$ papel principal lo desempeñan los bancos; sin embargo, el gobierno tiene un papel relevante al generar las condiciones que permiten el otorgamiento de crédito, principalmente a partir de medidas de política monetaria. Para la vertiente neochartalista, el papel principal lo desempeña el gobierno, con el manejo del gasto público (política fiscal), siendo la propuesta de esta vertiente la política del empleador de última instancia (ELR, por sus siglas en inglés), cuyo objetivo es garantizar el pleno empleo con estabilidad de precios.

Debido a que el ensayo tiene como objetivo presentar una alternativa de política económica que tenga como eje rector la reorientación del papel del Estado en la economía, resulta importante señalar qué tan factible es aplicar para el caso de México el programa de empleador de última instancia (ELR), el cual tiene como sustento teórico la vertiente poskeynesiana neochartalista. Dicha corriente considera que el dinero es endógeno y que el gobierno, al tener la facultad de establecer lo que se acepta como dinero, genera una demanda por el dinero que emite. ${ }^{4}$ El banco central es el banco del gobierno; por tal razón, para que el gobierno gaste no es necesario que exista ingreso previo, ya que siempre gasta abonando reservas al sistema bancario. Por tanto, la función de los impuestos, así como las operaciones de mercado abierto (instrumento de política monetaria), es drenar el exceso de reservas que genera el gasto gubernamental. Si el gobierno no drena el exceso de reservas, propicia una reducción de la tasa de interés, afectando con ello los objetivos de la política monetaria.

Los gobiernos de los países que emiten una moneda soberana son capaces de inyectar dinero a la economía, sin generar con ello un aumento de la inflación y de las tasas de interés, siempre y cuando el exceso de reservas creado en el sistema bancario sea drenado mediante el pago de impuestos y las operaciones de mercado abierto (venta de bonos).

Para poder instrumentar un programa ELR es necesario que el país emita moneda soberana y que tenga un régimen de libre flotación. "El objetivo principal (de dicho programa) es alcanzar el pleno empleo con estabilidad de precios. Otros objetivos complementarios incluyen la reducción de la pobreza, el mejoramiento de la infraestructura pública, la promoción de la producción interna para el consumo;

${ }^{3}$ Para más información sobre este enfoque poskeynesiano respecto al financiamiento de la inversión se puede consultar Arestis (2005), Moore (2006), Piégay y Rochon (2006), Smithin (2005) y Lavoie (2004).

4 "En todas las economías modernas el gobierno define el dinero, eligiendo lo que aceptará como pago de impuestos. Una vez que ha ordenado a los ciudadanos que paguen los impuestos en dinero, los ciudadanos deben obtener dinero para pagar esos impuestos" (Wray, 2006, p. 17.) 
y la provisión de servicios públicos" (Wray, 2007, p. 236). El gobierno debe decidir en qué trabajo va a contratar a los desempleados y fijarles un sueldo. Por lo general, dichos programas contratan a personas en actividades terciarias, como pueden ser guarderías, cuidado de los niños, limpieza y cuidado de las áreas verdes, becas para estudiar, etcétera. El salario que pague el gobierno debe ser inferior al del sector privado; por tal razón, dicho programa no reduce la mano de obra de las empresas privadas, ya que simplemente contrata a la gente que quiere trabajar, disminuyendo con ello el desempleo involuntario, mismo que debe llegar a ser cero. ${ }^{5}$

El gasto público que se utiliza para los programas de ELR debe ser financiado por la creación de dinero y el salario debe ser fijo. Se estipula que dichos programas permiten alcanzar el pleno empleo con estabilidad de precios, ya que se elimina el desempleo involuntario, sin producir presiones inflacionarias, debido a las siguientes razones: el salario es fijo en el nivel básico de remuneración (por lo general es el salario mínimo legal) y el pleno empleo (la desaparición del desempleo involuntario) no provoca que los trabajadores pidan mayores salarios, porque los empresarios siempre pueden encontrar mano de obra en los programas ELR (Wray, 2006, pp. 238-242).

Dichos programas no sólo son una propuesta teórica, pues se han instrumentado en Argentina (Programa Jefes y Jefas del Hogar) e India, consiguiendo a partir de su puesta en marcha una disminución del desempleo y un mayor crecimiento económico. Sería pertinente preguntarse si es factible que en México se instrumente un programa ELR. Para los poskeynesianos la respuesta es afirmativa, ya que México es una nación con moneda soberana, régimen de cambio flotante y un alto desempleo; no obstante, algunos investigadores mexicanos han subrayado que en México no es viable, ya que las cartas de intención que se tienen firmadas con el FMI impiden tener déficit fiscales, por considerarlos inflacionarios.

No obstante, es importante destacar que las ventajas que un programa ELR puede generar en la economía mexicana, como son pleno empleo, mayor crecimiento, estabilidad de precios, disminución de la pobreza, mejor distribución del ingreso, etc. son mayores que el costo político que genera su instrumentación. Por tanto, en México es económicamente viable y factible la implantación de un programa ELR que mejore las condiciones de vida de los mexicanos.

\footnotetext{
${ }^{5}$ Otros neochartalistas que coinciden con este enfoque se encuentran principalmente en Forstater (2001) y Tcherneva (2005)
}

\section{La reorientación de la función del Estado}

A continuación se presentan los aspectos relevantes sobre la reorientación de la función del Estado que deben de considerarse a fin de avanzar en la propuesta de que éste sea uno de los sectores que dinamice el crecimiento de la economía y el combate al desempleo para que en el mediano plazo permitan mejorar las condiciones de vida de la población y reducir la desigualdad social que produce la aplicación del modelo neoliberal.

En primera instancia, el planteamiento que realizamos como la reorientación de la función del Estado en la economía se enfoca principalmente hacia reconocer que las acciones tomadas a mediados de la década de los ochenta para reducir el papel del Estado en la economía y dejar en manos del mercado la determinación de los precios y la asignación eficiente de los factores de producción, como lo proponían los teóricos de la represión financiera, no han brindado resultados de eficiencia económica y de un proceso de mayor competitividad de la economía mexicana. Por el contrario, los resultados han sido, por ejemplo, en el caso del sector financiero de la economía, la existencia actual de un sector bancario oligopolizado y mayoritariamente propiedad del capital extranjero.

Esto mismo ha sucedido en otros sectores de la producción real de la economía, en donde la apertura del mercado ha redundado en la baja de nuestro sector exportador, el aumento de las importaciones y el cierre de varias empresas (sobre todo pequeñas y medianas) que han significado una importante reducción en la generación de empleos formales en nuestro país. Así, se ha expandido de manera importante un sector informal del empleo en crecimiento que ha provocado la reducción de ingresos fiscales y el fomento del contrabando técnico en la economía, pese a los objetivos de mantener finanzas públicas sanas o en equilibrio.

Por otra parte, las condiciones de fomentar la inversión pública como mecanismo para incrementar el empleo en la economía no se han dado; por tanto, el Estado no ha podido actuar de manera contracíclica para elevar el crecimiento de nuestra economía en los últimos años, dados los parámetros que ha impuesto el modelo neoliberal.

Por su parte, las aportaciones de los nuevos keynesianos (especialmente Stiglitz, 2006) señalan que es fundamental la redistribución del ingreso por parte del Estado debido a la existencia de las "fallas de mercado". Es decir, el primer teorema del bienestar económico señala que la economía es eficiente en términos de Pareto, únicamente bajo ciertas circunstancias o condiciones de mercados competitivos y eficientes. Sin embargo, existen circunstancias en que la econo- 
mía no es Pareto-eficiente, debido a la existencia de fallas como son, por ejemplo, la existencia de monopolios, externalidades, bienes públicos, mercados incompletos, fallas en la información, desempleo e inflación. Entonces, el segundo teorema del bienestar económico nos dice que si la distribución del ingreso producida por el mercado no es socialmente aceptable, en respuesta todos los gobiernos necesitan redistribuir las dotaciones iniciales, con el fin de desaparecer los efectos nocivos que generan estas "fallas de mercado" y obtener así una distribución del ingreso socialmente aceptable.

En este contexto, si bien es cierto que en el sector bancario las condiciones de oligopolio existían antes del proceso de desregulación del sector financiero en la economía de nuestro país, también es cierto que estas condiciones de mercado se han profundizado, distorsionando aún más el funcionamiento del sector bancario en nuestro país; por tanto, el concepto de reorientación de la función estatal en la economía va en el sentido de que es necesario que el Estado mexicano reconozca que el mercado por sí mismo no es el mejor mecanismo en la distribución y uso de los recursos.

En consecuencia, se hace necesario replantear la función del Estado en la economía mediante acciones tendientes a mejorar el funcionamiento del mercado en el sentido de establecer, para el caso del sector financiero y en particular del bancario, límites al abuso de márgenes financieros altos por parte de la banca comercial, fijando máximos aceptables a las tasas de interés activas que lleven a sector bancario a una demanda de crédito competitiva de acuerdo con las condiciones del mercado internacional.

Asimismo, es necesario fomentar el financiamiento bancario al sector real de la economía a partir del sector bancario y promover así la inversión productiva y, en consecuencia, el ahorro y empleo derivados de una reducción en el costo del crédito. También es necesario fortalecer a las instituciones de banca de desarrollo mediante la promoción del crédito al sector industrial con potencial de crecimiento en el empleo a costos preferenciales, a fin de fortalecer también a las empresas orientadas a la atención de la demanda interna de nuestro país.

Otra de las acciones necesarias, dadas las condiciones oligopólicas del sector bancario, es la de establecer mínimos a depósitos de la banca comercial en el Banco de México a fin de que en lugar de seguir emitiendo cantidades importantes de valores gubernamentales - que no hacen más que favorecer la rentabilidad de la banca comercial-, promueva a costo de Cetes el financiamiento de sectores económicos con rezago, como es el caso del sector agropecuario.

Como podemos observar, el planteamiento de la reorientación de la función del Estado en la economía no va en el sentido de regresar al viejo paradigma de una mayor participación del Estado en la economía, sino de que éste se oriente a promover acciones tendientes a mejorar la regulación en el funcionamiento del mercado nacional mediante acciones negociadas con el sector privado de la economía, buscando mejorar las condiciones de producción, competitividad de la industria, eficiencia en el funcionamiento del sector financiero y bancario, promoción del empleo y, finalmente, mejoramiento de las condiciones sociales y de vida de la población mexicana. Lo anterior, dado que el modelo económico basado totalmente en el funcionamiento del mercado no ha resultado, y por lo contrario, ha elevado las condiciones de pobreza, de deterioro social, falta de seguridad social además de haber incrementado el desempleo y la desigualdad en el desarrollo regional entre el norte y sur de nuestro país.

Por tanto, el concepto de reorientación en la función del Estado en la economía no sólo comprende acciones de carácter económico, sino también de tipo social, que tiendan a ser acordes a las condiciones de crecimiento con una mejora en la distribución del empleo y en la distribución de la riqueza generada actualmente en la economía con la optimización, además, de una reforma fiscal integral con carácter social y no solamente en beneficio de los que más ganan o bien de que su objetivo sea en sí mismo el aumento de los ingresos tributarios, sino que dicha reforma debe tener un carácter distributivo y social en el aumento de los ingresos fiscales del gobierno federal.

No avanzar en esta perspectiva puede llevar a nuestro país a incrementar el descontento social y político que actualmente se ha empezado a reflejar en varios países de América Latina, en donde sus sociedades ya han empezado a optar por la elección de gobiernos con visiones sociales, en donde el Estado desempeña un papel importante en el proceso de negociación a fin de mejorar las condiciones económicas de sus sociedades, como es el caso reciente del gobierno boliviano que llevó a cabo la nacionalización de la industria petrolera.

Finalmente, considerando la visión poskeynesiana de la importancia del financiamiento al sector real de economía para promover el ingreso, el empleo y en consecuencia el ahorro, ésta debe ser promovida a partir del nuevo papel del Estado mediante su reorientación ya que, como se ha observado en más de veinte años, el mercado no es el mejor asignador ni de precios ni de recursos, sobre todo en una economía emergente; por tal razón, resulta muy importante que el Estado establezca acciones tendientes a la regulación, a fin de reducir las imperfecciones que existen en mercados expuestos a la globalización, sin ningún respaldo del Estado, en promoción de la inversión y el empleo en nuestro país.

Asimismo, se deberá promover el gasto de inversión federal, estatal y municipal, sin que ello provoque desequilibrios en las finanzas públicas, sino más 
bien haciendo un uso racional de los recursos del gobierno federal con la reducción en el crecimiento del endeudamiento público interno y ampliando el margen de maniobra del gasto público no programable que permita incrementar el gasto de inversión, junto con regulaciones específicas a las tasas activas, a la creación de fondos de inversión en manos del Banco de México, al logro de un uso productivo del fondo de ahorro para el retiro de los trabajadores mediante la regulación específica a las Siefores y Afores, que actualmente están en manos del sector bancario.

\section{Conclusiones}

En esta investigación se analizan los cambios que ha padecido la política de gasto de desarrollo social en México, en el contexto del modelo neoliberal. Se señala que con el modelo de sustitución de importaciones prevalecían políticas económicas de corte keynesiano que apoyaban la intervención del Estado en la economía, empleando el gasto gubernamental como motor de crecimiento económico. Por tal razón, en las políticas públicas, el gasto social, el gasto en infraestructura y el gasto en inversión tenían una fución relevante.

En el modelo neoliberal, regido por políticas de corte monetarista, se considera que los mercados funcionan de forma eficiente siempre y cuando se les deje actuar libremente, sin intervención gubernamental. Los ejes de la política neoliberal se sustentan en la liberalización financiera, la liberalización comercial y la liberalización económica. Las políticas neoliberales en América Latina fueron fomentadas e impulsadas a partir de lo que se conoce como el Consenso de Washington, cuyos principales exponentes afirmaban que con la instrumentación de las políticas neoliberales se lograría: $a$ ) aumentar el nivel de competencia b) combatir la inflación, y c) consolidar un crecimiento económico estable que generara fuentes de empleo.

No obstante, en México, los niveles de pobreza, de desempleo y de desigualdad del ingreso se han agudizado a partir de la entrada en vigor del modelo neoliberal, propiciando así la inestabilidad en el crecimiento económico, y todo ello se traduce en un proceso de desarrollo que deteriora las condiciones de vida de la población.

Frente a tal panorama este ensayo presentó una propuesta de reorientación del Estado para fortalecer su carácter anticíclico, mediante la instrumentación de políticas públicas, como de infraestructura y de desarrollo, que fortalezcan al mercado interno y promuevan la creación de empleos. Por tanto, se expone la instrumentación de una política de gasto público que no se convierta en un paliativo para disminuir la pobreza y la desigualdad del ingreso, sino más bien en una política pública que sea un motor de crecimiento.

En este contexto, se analizó la viabilidad que puede tener en México la política de empleador de última instancia (ELR) propuesta por los poskeynesianos, ya que dichos programas han sido exitosos en los países en los que han sido instrumentados, como Argentina e India.

Es importante señalar, por un lado, que un programa de empleador de última instancia puede crear efectos positivos en la economía mexicana, como pleno empleo, mayor crecimiento, estabilidad de precios, disminución de la pobreza, mejor distribución del ingreso, etc., y, por el otro, que dichos beneficios son mayores que el costo político que genera su instrumentación. Por tanto, en México es económicamente viable y factible la instrumentación de un programa ELR que mejore las condiciones de vida de los mexicanos.

Por reorientación de la función del Estado en la economía entenderemos no el regreso a las prácticas de intervención estatal derivadas del planteamiento keynesiano, sino su participación en la regulación de la actividad económica.

En este sentido, es necesario el mantener sectores estratégicos en poder del Estado a fin de evitar presiones inflacionarias o insuficiencia de oferta por efecto de la transferencia de su propiedad al sector privado; asimismo, que el Estado proporcione servicios de salud pública, seguridad y educación básica, renglones que no pueden quedar en manos del sector privado de la economía debido a que este sector no tendría la capacidad de atención de estos servicios.

Finalmente, con la intención de corregir las imperfecciones del mercado que dan lugar a la constitución de monopolios, oligopolios y otras formas de mercado que impiden la formación de precios y asignación de recursos eficientes, el Estado está obligado a establecer normas y acciones de regulación que busquen reducir estos desajustes. Por ejemplo, ante el proceso de globalización y desregulación del sector financiero mexicano que ha dado lugar a la conformación de un mercado oligopólico, es necesario establecer normas que regulen de manera directa los niveles de tasas de interés tanto pasivas como activas, a fin de que el sector financiero se convierta en un promotor del financiamiento al sector productivo y, por ende, del desarrollo económico de largo plazo en México.

Asimismo, frente a una generación insuficiente de empleos en la economía mexicana debido a los problemas que enfrentan las pequeñas y medianas empresas, es necesario establecer programas de apoyo financiero a partir de la banca de desarrollo y del fomento de la inversión pública, y posibilitar un efecto multiplicador de la inversión que mejore las expectativas de inversión del sector privado en la generación de empleos. 
Por otra parte, es necesario establecer lineamientos en las políticas públicas que si bien no logran el óptimo de Pareto busquen con su implantación disminuir la brecha cada vez más grande entre ricos y pobres en la sociedad mexicana mediante la aplicación de un programa nacional de apoyo a la población con niveles de ingresos inferiores a los dos salarios mínimos mensuales, con el fin de mejorar su calidad y expectativas de vida, sobre todo en la región sur de nuestro país.

\section{REFERENCIAS BIBLIOGRÁFICAS}

Arestis, Philip, y Sawyer Malcolm (2005), "Aggregate demand, conflict and capacity in the inflationary process". Cambridge Journal of Economics, núm. 29, pp. 959-974. Ayala Espino, José (2004), Mercado, elección pública e instituciones. Una teoría de las teorías modernas del Estado: México: unAm-Porrúa.

Girón González, Alicia, y Eugenia Correa (2002), Crisis y futuro de la banca en México. México: unam-Porrúa.

Ibarra, David (2006), La reconfiguración económica internacional. México: UNAM.

Keynes, J. M. (1974), Teoría general de la ocupación, el interés y edinero. México: FCE.

Lavoie, Marc (2004), La economía postkeynesiana. Un antídoto del pensamiento único. Barcelona: Icaria.

Martínez Marca, José Luis (2006), "La reorientación del papel del Estado en la economía". Documento en CD. México: Fes-unam.

_ (2007), "La política monetaria y banca comercial en México (el caso del margen financiero) 1995-2005". Documento en proceso de impresión. México: unam.

Moore, Basil (2006), “La endogeneidad de la oferta de dinero: ¿se fija el precio o la cantidad de reservas? En Piégay, Pierre, y Louis-Philippe Rochon (comps.), Teorías monetarias poskeynesianas. Akal: Madrid, pp. 46-53.

Piégay, P., y Louis-Philippe Rochon (2006), “Introducción. Dinero endógeno y economías monetarias de producción: la aportación de las teorías monetarias poskeynesianas". En Piégay, P., y L. Rochon (comps.), Teorías monetarias poskeynesianas.Akal: Madrid.

Smithin, y Eric Kam (2005), "Notas sobre el dinero, crédito y banca en el proceso de desarrollo económico". Economía Informa, núm. 337, pp. 6-16.

Stiglitz, Joseph (2006), Economics of the Public Sector. Nueva York: W. W. Norton y Company.

Tcherneva, Paulina (2005), "The art of Job Creation; promises and problems of the Argentinean experience". Special Report of CFEPS (Centro para el Pleno Empleo y Estabilidad de Precios), 19 de septiembre.
Tello, Carlos (2007), Estado y desarrollo económico: México 1920-2006. México: unAm. Wray Randal, Larry (2000), "Keynes y el pleno empleo: una lectura contemporánea". Comercio Exterior, 50 (12).

_ (2005), "Una estructura monetaria y fiscal para la estabilidad económica: un enfoque friedmaniano". En Eugenia Correa et al. (coords.), Consecuencias financieras de la globalización. México: UNAm/IIEc/Porrúa.

_ (2006), "El enfoque poskeynesiano del dinero". En Pierre Piégay y Rochon (comps.), Teorías monetarias poskeynesianas. Madrid: Akal.

- (2006 a), El papel del dinero hoy: la clave del pleno empleo y la estabilidad de precios. México: unam-Facultad de Economía.

_ (2007), "Entendiendo la política económica en régimen cambiario de libre flotación”. En Guadalupe Mántey et. al., Políticas macroeconómicas para países en desarrollo. México: Porrúa. 


\section{Colaboradores}

\section{Graciela Gutman}

Licenciada en economía política, actualmente se desempeña como profesora en la Universidad Nacional de Buenos Aires, en Argentina; es vicedirectora e investigadora principal del Centro de Estudios Urbanos y Rurales-Consejo Nacional de Investigaciones Científicas (CEUR-CONICET).

\section{Pablo Lavarello}

Doctor en economía. Actualmente se desempeña como profesor de la Universidad de Buenos Aires en Argentina e investigador del CEUR-CONICET.

José Luis Martínez Marca

Doctor en economía, maestro en economía financiera y licenciado en economía por la UNAM. Sus líneas de investigación son: área teórico-metodológica en políticas públicas como: política monetaria y banca comercial, así como en finanzas públicas y moneda y finanzas. Es miembro del Sistema Nacional de Investigadores del CONACyT, nivel I. Actualmente se desempeña como profesor titular B definitivo de tiempo completo del Posgrado en Economía de la FES-Aragón UNAM, en las materias de Moneda y finanzas II y Finanzas públicas, y también es profesor de asignatura B definitivo en las materias de Economía financiera y estructura económica en la licenciatura en economía.

Alejandro Bello

Doctor en organización de empresas. Ha sido profesor visitante en la Universidad Autónoma de Barcelona. Sus líneas de investigación se enfocan al análisis económico y estratégico de sectores energéticos, principalmente de hidrocarburos líquidos. Algunas de sus investigaciones más recientes han sido presentadas 
en congresos nacionales e internacionales y publicados en prestigiadas revistas como son Energy Policy, Revista de Economía Aplicada o International Journal of Operations and Production Management. Actualmente se desempeña como profesor en el Departamento de Gestión de Empresas de la Universidad Pública de Navarra, España.

\section{Sandra Cavero}

Se ha desempeñado como profesora en la Universidad Pompeu Fabra y profesora visitante en la Universidad de Purdue; también como directora del Servicio de Planificación Estratégica y Evaluación de Política de Asistencia Social en el gobierno de Navarra, España. Orienta sus líneas de investigación a la estrategia y el análisis económico de las organizaciones. Su investigación corriente dirige el impacto de mercados desregulados sobre la estrategia de negocio y el bienestar. Sus investigaciones han sido publicadas en revistas de prestigio como International Journal of Research in Marketing o Energy Policy. Actualmente se desempeña como profesora titular de la Dirección Estratégica en la Universidad Pública de Navarra, España.

\section{Leonardo Egidio Torre}

Doctor en economía por la Universidad de California, Los Ángeles, y tiene una maestría en economía por la Universidad de Cincinnati. Se ha desempeñado como coordinador general de asesores en la Subsecretaría de Negociaciones Internacionales de la Secretaría de Economía, y como director general adjunto de Estudios Económicos Internacionales de la SHCP. Sus campos de especialización son Finanzas Internacionales, Desarrollo Económico y Organización Industrial. En la Facultad de Economía de la UANL pertenece al cuerpo académico consolidado de la Organización Industrial y Regulación PROMEP-SEP. Actualmente es profesor de tiempo completo en la Facultad de Economía de la UANL. Es miembro del Sistema Nacional de Investigadores nivel I y gerente de Estudios Económicos de Cemex-México.

\section{María Elena Lavín}

Maestra en economía industrial por la Universidad Autónoma de Nuevo León, especializada en el área de Finanzas corporativas y Finanzas internacionales. Es contadora pública y auditora por la Universidad de Monterrey. Actualmente se desempeña como profesora auxiliar en el área Económico-Administrativa de la Universidad TEC Milenio y es auditor interna de Supermercados Internacionales H-E-B. 
